\title{
Resistência de União entre Dentes Artificiais e Resinas Acrílicas para Base Protética
}

\author{
Ana C. Pero, Juliê Marra, Cilene A. Polatto, Débora B. Barbosa, Marco A. Compagnoni \\ Departamento de Materiais Odontológicos e Prótese, FOAr - UNESP
}

Resumo: A resistência de união entre dente artificial e resina acrílica para base protética foi avaliada sob a influência de duas marcas comerciais de dentes artificiais (Biotone e Trilux) e de três resinas acrílicas, sendo duas polimerizadas convencionalmente em banho de água (QC-20 e Lucitone 550) e uma resina polimerizada por meio da energia de microondas (Acron-MC). As avaliações foram realizadas por meio de um ensaio mecânico de cisalhamento através da aplicação de uma carga perpendicular à interface dente-resina. Os resultados indicaram que, para a mesma resina, a resistência foi semelhante, independentemente do tipo de dente utilizado. Para os dois tipos de dente avaliados, as resinas Acron MC e Lucitone 550 apresentaram valores de resistência de união entre dente artificial e resina acrílica estatisticamente semelhantes e superiores em relação à resina QC-20.

Palavras-chave: Resina acrílica, resistência de união, dente artificial, microondas.

\section{Shear Bond Strength between Artificial Acrylic Resin Teeth and Denture Base Resins}

Abstract: Shear bond strength between artificial acrylic resin teeth and denture base resin was evaluated under the influence of two brands of artificial teeth (Biotone and Trilux) and three denture base resins. Two of these resins were conventionally polymerized in water bath (QC-20 and Lucitone 550), while the other was a microwave-polymerized resin (Acron-MC). The evaluations were done by means of a shear bond strength test with application of a perpendicular load to the interface tooth-resin. The results showed that, for the same denture resin, the strength was similar regardless of the tooth brand. Also, for the two evaluated brands of tooth, the resins Acron MC and Lucitone 550 presented similar shear bond strengths, which were statistically higher than for resin QC-20.

Keywords: Acrylic resin, shear bond strength, artificial tooth, microwaves.

\section{Introdução}

Os dentes artificiais de resina acrílica foram introduzidos na Odontologia em $1940^{[1]}$, apresentando como principais vantagens a estética e a facilidade de ajustes durante a confecção de próteses totais e removíveis. No entanto, as desvantagens desse tipo de dente são a baixa resistência ao desgaste e a susceptibilidade à fraturas e à falhas adesivas entre o dente artificial e a base protética de resina acrílica.

Fatores como o tipo e o tratamento da superfície do dente artificial utilizado, a fase de prensagem e as variáveis de processamento das resinas acrílicas ${ }^{[2-8]}$, bem como a presença de impurezas na interface dente-resina acrílica, o tipo de resina acrílica e a sua temperatura de polimerizaçãa ${ }^{[9]}$ podem interferir na união entre o dente artificial e a resina acrílica para base protética.

Assim como Polyzois \& Dahal ${ }^{[10]}$, Schneider et al. ${ }^{[11]} \mathrm{e}$ Takahashi et al. ${ }^{[7]}$ verificaram que a resina acrílica polimerizada em banho de água forneceu uma maior resistência de união entre dente artificial e resina acrílica, quando comparada à uma resina acrílica polimerizada por meio de energia de microondas. No entanto, Yamauchi et al. ${ }^{[12]}$ e Geerts \& Jooste $^{[6]}$, em estudos semelhantes, observaram que os gru- pos polimerizados em microondas forneceram resultados mais favoráveis.

O método de polimerização de resinas acrílicas por meio da energia de microondas em Odontologia foi introduzido por Nishii ${ }^{[13]}$ há cerca de 40 anos. Esse método apresenta como principal vantagem a grande economia de tempo na polimerização da resina acrílica. Esse rápido aquecimento ocorre em função de um campo eletromagnético de alta freqüência $(2450 \mathrm{MHz})$ gerado no interior do forno de microondas, resultando em inúmeras colisões entre as moléculas do monômero ${ }^{[14]}$.

No entanto, o excesso de aquecimento promovido por altas potências no forno de microondas, juntamente com a exotermia da reação de polimerização da resina acrílica podem causar porosidade indesejável neste material ${ }^{[15,16]}$. A dificuldade de se controlar a temperatura no interior do microondas pode levar à ebulição do monômero durante o processo de polimerização, e resultar na formação de poros na resina acrílica. A localização dos poros na interface denteresina poderia promover uma falha de união entre estes dois materiais, como tentaram explicar Polyzoiz \& Dahal ${ }^{[10]}$ ao encontrarem uma menor resistência de união entre dentes artificiais e a resina acrílica polimerizada por microondas.

Autor para correspondência: Marco A. Compagnoni, Departamento de Materiais Odontológicos e Prótese, Faculdade de Odontologia de Araraquara, UNESP, Rua Humaitá 1680, CEP: 14801-903, Araraquara, SP, Brasil. E-mail: compagno@foar.unesp.br. 
Com isso, o objetivo da presente pesquisa foi avaliar a resistência de união entre dois tipos de dente artificial de resina acrílica com duas resinas acrílicas para base protética por meio de ensaio mecânico de cisalhamento. Existiram duas hipóteses para esse estudo. A primeira foi que, para uma mesma resina acrílica, a resistência de união entre o dente artificial e resina acrílica não é influenciada pelo tipo de dente. A segunda é que, para o mesmo tipo de dente, a resistência de união não depende do tipo de resina acrílica utilizado.

\section{Experimental}

\section{Material e método}

Foi avaliada a resistência de união entre dois dentes artificiais de resina acrílica (Biotone/ $30 \mathrm{M}$ - Dentsply Ind. e Com. Ltda - Rio Janeiro, RJ, Brasil; e Trilux/ M5 - RuthiBras Imp. Exp. E Com. De Odontológicos Ltda - Pirassununga, SP, Brasil ) e três resinas acrílicas para base protética (Tabela 1). Dez espécimes foram confeccionados para cada um dos grupos estudados (Tabela 2).

\section{Obtenção do espécime}

O espécime correspondeu ao conjunto de um dente artificial embutido no polímero da resina acrílica autopolimerizável (Jet) e a resina acrílica polimerizada nos ciclos propostos acima, posicionada perpendicularmente a esse dente.

Foi selecionado o $1^{\circ}$ molar superior direito de 2 tipos de dentes artificiais de resina acrílica (Trilux e Biotone). Previamente, a superfície cervical do dente artificial foi lixada com lixas d'água (Norton; Saint-Gobain Abrasivos Ltda, Vinhedo, SP, Brasil) seqüenciais de diferentes granulações (320, 400 e 600) em uma politriz (Arotec Ind. e Com. Ltda, Cotia, SP, Brasil) a $300 \mathrm{rpm}$ até atingir-se uma superfície plana (Figura 1). Esse procedimento permitiu que a interface dente artificial-resina acrílica ficasse plana, evitando áreas de concentração de tensão no dente ou na resina durante o ensaio de cisalhamento com o cinzel na interface.

Em seguida, utilizando-se uma embutidora (Arotec Ind. e Com. Ltda, Cotia, SP, Brasil), cada dente foi embutido em polímero da resina acrílica autopolimerizável (Jet, Artigos Odontológicos Clássico Ltda, São Paulo, SP, Brasil). A sequiência laboratorial para o embutimento do dente artificial encontra-se descrita na Figura 2. O embutimento do dente favoreceu o seu acabamento com lixas na politriz (Figura 3) e permitiu a fixação do espécime em uma matriz para a realização do ensaio de cisalhamento (Figura 4)

Um molde em silicone (Zetalabor, Zhermack S.A. - Rovigo - Itália) foi confeccionado com o auxílio de uma matriz metálica (Figura 5) que apresentava centralmente a réplica das dimensões da resina acrílica a ser processada sobre o dente. Essas dimensões seguiram as normas da International Organization for Standardization ${ }^{[17]}$, especificação 10477. O silicone foi condensado no interior dessa matriz e após a sua polimerização, o molde obtido foi fixado com adesivo

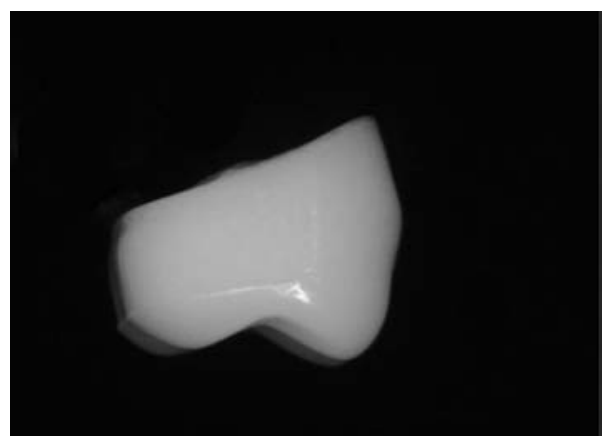

(a)

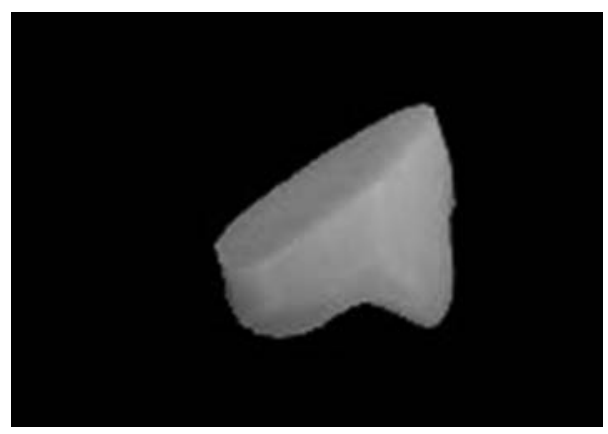

(b)

Figura 1. Dente artificial a) antes; e b) após lixamento da superfície cervical na politriz.

Tabela 2. Grupo/resina acrílica/ciclos de polimerização e dente artificial

\begin{tabular}{cll}
\hline Grupo & Resina acrílica & Dente artificial \\
\hline $\mathrm{A}_{1}$ & Acron-MC & Biotone \\
$\mathrm{A}_{2}$ & Acron-MC & Trilux \\
$\mathrm{Q}_{1}$ & QC-20 & Biotone \\
$\mathrm{Q}_{2}$ & QC-20 & Trilux \\
$\mathrm{L}_{1}$ & Lucitone 550 & Biotone \\
$\mathrm{L}_{2}$ & Lucitone 550 & Trilux \\
\hline
\end{tabular}

Tabela 1. Resinas acrílicas utilizadas no estudo.

\begin{tabular}{|c|c|c|c|c|}
\hline Resina acrílica & Fabricante & Tipo & Ciclo de polimerização* & Lote No. \\
\hline Acron MC & $\begin{array}{l}\text { GC Lab Technologies, Inc. - } \\
\text { Alsip, Illinois, U.S.A. }\end{array}$ & $\begin{array}{l}\text { - Específica para polimeriza- } \\
\text { ção em microondas }\end{array}$ & $3 \mathrm{~min}$ a $500 \mathrm{~W}$ & 0303141 \\
\hline Lucitone 550 & $\begin{array}{l}\text { Dentsply Ind. e Com. Ltda - } \\
\text { Rio Janeiro, RJ, Brasil }\end{array}$ & $\begin{array}{l}\text { Específica para polimeriza- } \\
\text { ção em banho de água }\end{array}$ & $90 \min$ a $73{ }^{\circ} \mathrm{C}$ e $30 \min$ a $100^{\circ} \mathrm{C}$ & 186120 \\
\hline QC 20 & $\begin{array}{l}\text { Dentsply Ind. e Com. Ltda - } \\
\text { Rio Janeiro, RJ, Brasil }\end{array}$ & $\begin{array}{l}\text { Específica para polimeriza- } \\
\text { ção em banho de água }\end{array}$ & Água em ebulição por 20 min & 6168 \\
\hline
\end{tabular}

*Ciclos propostos pelos fabricantes das respectivas resinas acrílicas. 


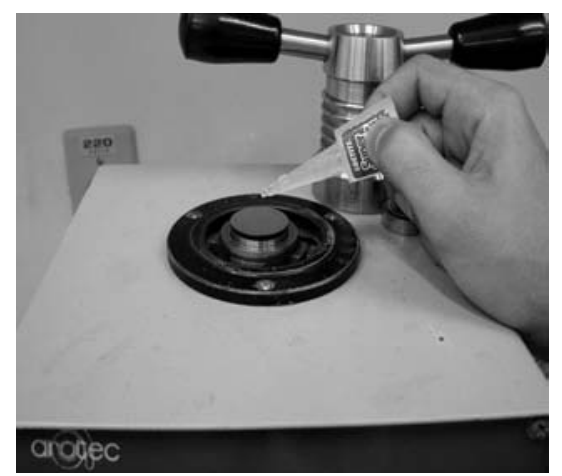

(a)

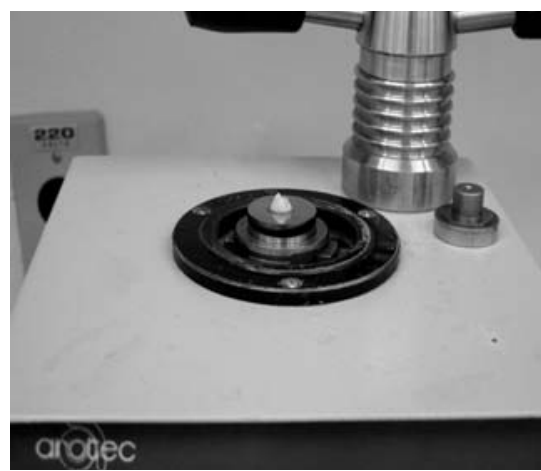

(b)

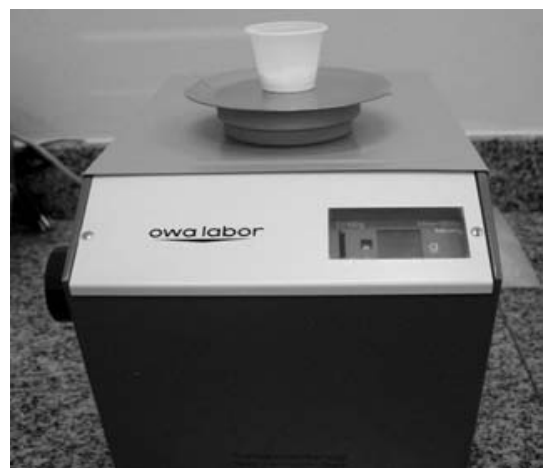

(c)

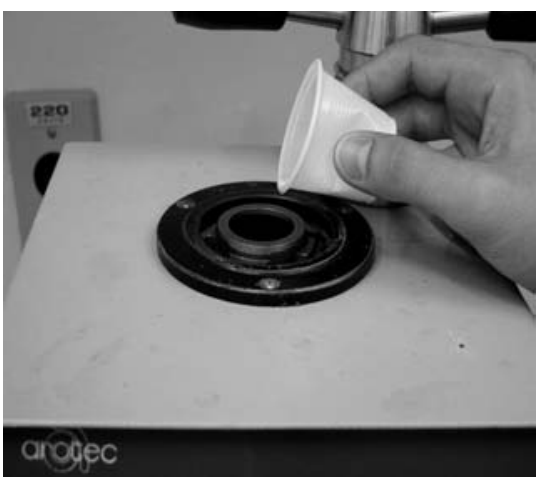

(d)

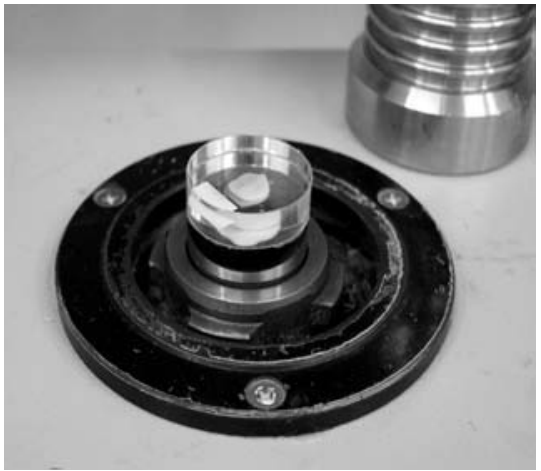

(e)

Figura 2. Embutimento do dente artificial: a) aplicando uma gota de adesivo instantâneo na porção central da base do cilindro metálico da embutidora onde será fixado o dente; b) fixação do dente na superfície do cilindro metálico; c) pesagem do polímero da resina autopolimerizável (7,0 g.); d) Após abaixar o cilindro metálico onde está fixado o dente, inserção do polímero; e e) depois de fechar hermeticamente a abertura da embutidora e aguardar cerca de 15 minutos sob pressão de $150 \mathrm{Kgf} / \mathrm{cm}^{2}$ ( 7 minutos em alta temperatura e mais 7 para o resfriamento do espécime), o cilindro metálico onde foi fixado o dente é suspenso com o dente já embutido.

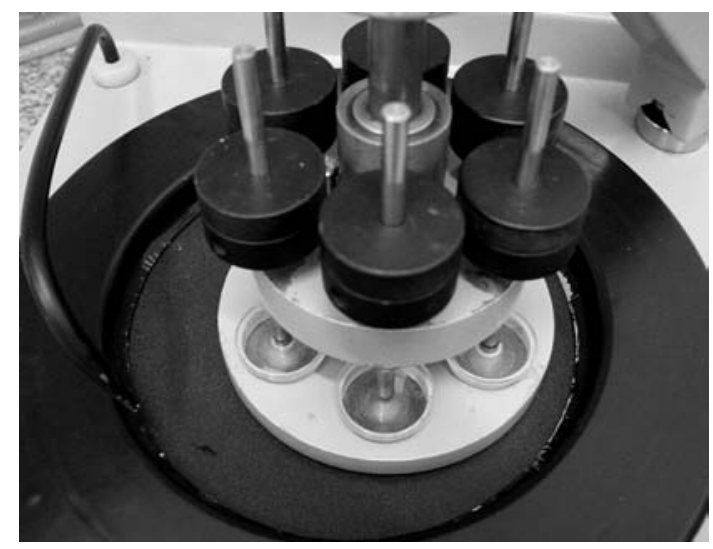

(a)

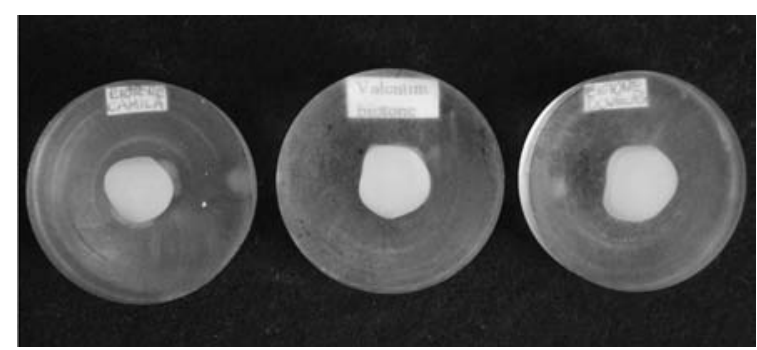

(b)

Figura 3. Acabamento dos dentes embutidos: a) amostra de dentes embutidos posicionados nas lojas do braço da politriz; e b) dentes embutidos já lixados.

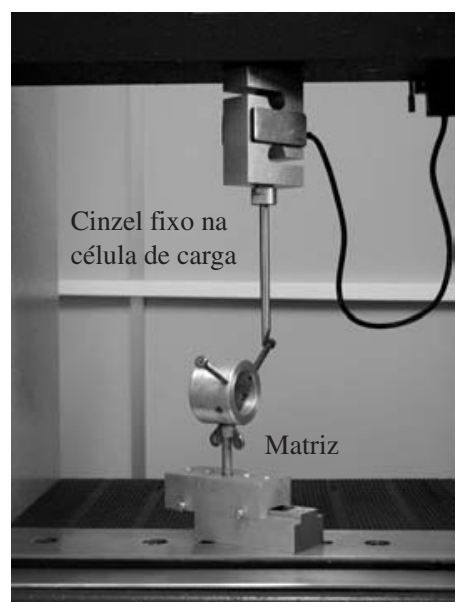

Figura 4. Matriz de fixação do espécime para o ensaio mecânico de cisalhamento.

instantâneo sobre a superfície do dente embutido (Figura 6). A obtenção desse molde permitiu a condensação e processamento da resina acrílica sobre a superfície do dente artificial, e facilitou o procedimento de desinclusão do espécime.

Por tratar-se de resinas acrílicas polimerizáveis pela ação do calor, há necessidade de inclusão do conjunto molde/dente embutido em gesso pedra tipo III no interior de muflas. Para a polimerização em microondas utilizou-se mufla plástica e para polimerização em banho de água quente, mufla metáli- 


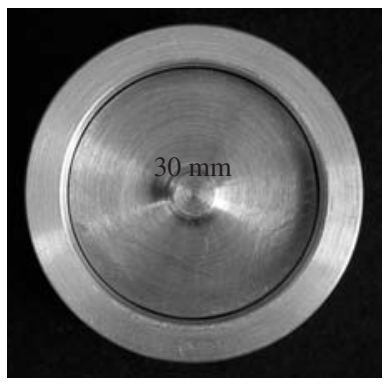

(a)

Figura 5. Matriz metálica.

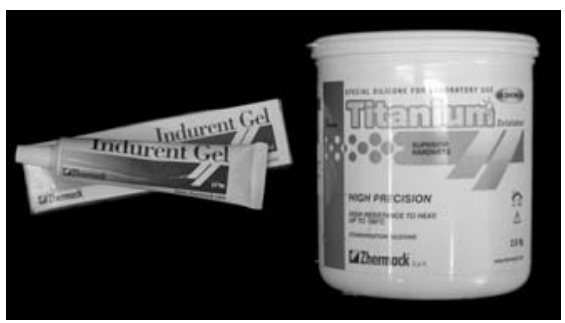

(a)

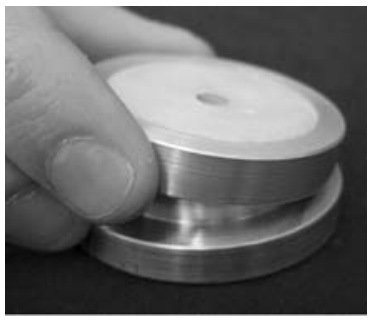

(e)

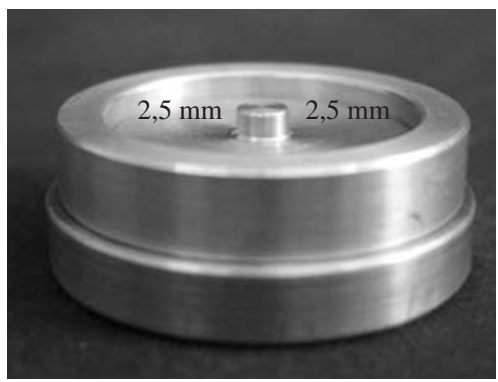

(b)

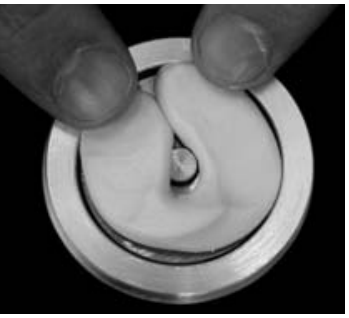

(b)

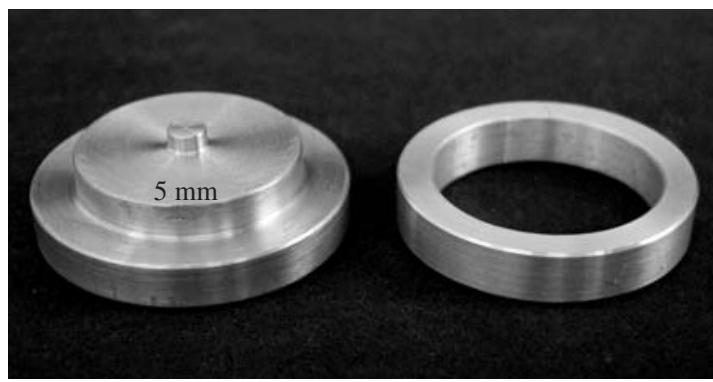

(c)

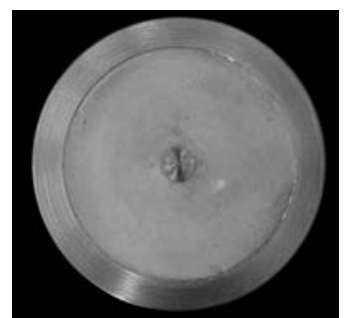

(c)

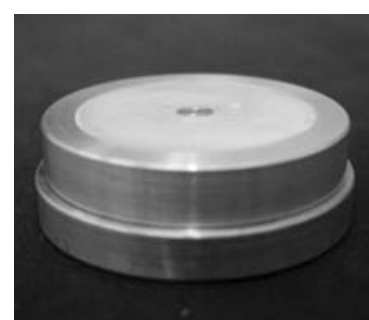

(d)

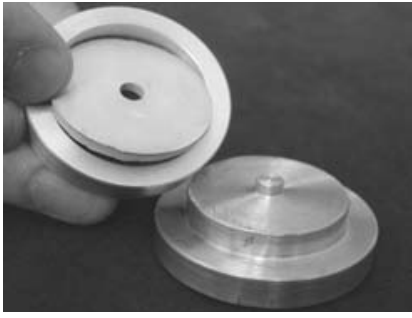

(f)

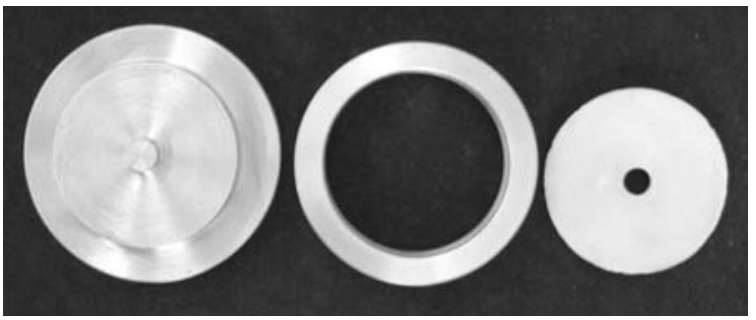

(g)

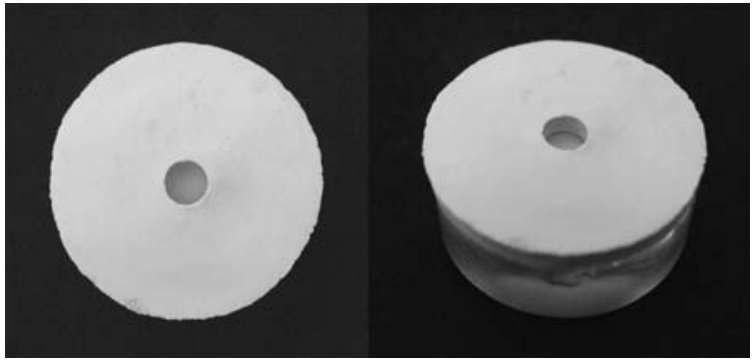

(h)

Figura 6. Obtenção do molde em silicone: a) silicone para inclusão; b) condensação do silicone no interior da matriz; c) e d) silicone já condensado; e) e f) remoção do molde do interior da matriz; g) matriz ao lado do molde pronto; e h) molde em silicona fixado sobre o dente embutido, evidenciando a centralização do orifício sobre o dente artificial.

ca. Previamente ao vazamento do gesso no interior da mufla, o orifício do molde foi preenchido com uma pequena quantia de silicone para impedir a penetração de gesso no seu interior no momento da inclusão.

Após a presa do gesso da inclusão, a mufla foi aberta e o silicone do interior do orifício foi cuidadosamente removido com uma sonda exploradora. As resinas acrílicas em forma de pó (polímero) e líquido (monômero) foram proporcionadas de acordo com as recomendações dos fabricantes. Ao atingirem a fase plástica, as resinas foram condensadas no interior do orifício do molde, a mufla foi fechada e levada em uma prensa hidráulica para a prensagem lenta e gradual das resinas ${ }^{[18]}$. Após 30 minutos sob a prensa para melhor es- coamento da resina no interior do molde e para maior aglutinação do pó no líquido, as resinas foram polimerizadas nos ciclos propostos na Tabela 2 .

Após a polimerização, as muflas foram resfriadas sobre bancada por uma noite para não ocorrer distorção da resina acrílica. As muflas foram abertas e o gesso foi removido da mufla para a desinclusão dos espécimes (Figura 7a). Em seguida, o silicone foi removido cuidadosamente para a exposição da resina acrílica já polimerizada sobre a superfície do dente artificial (Figura 7b). Os espécimes (Figura 7c) foram mantidos em estufa à $37^{\circ} \mathrm{C}$ imersos em água destilada por $50 \pm 2$ horas previamente à realização do ensaio mecânico. Esse procedimento seguiu as normas da International Orga- 


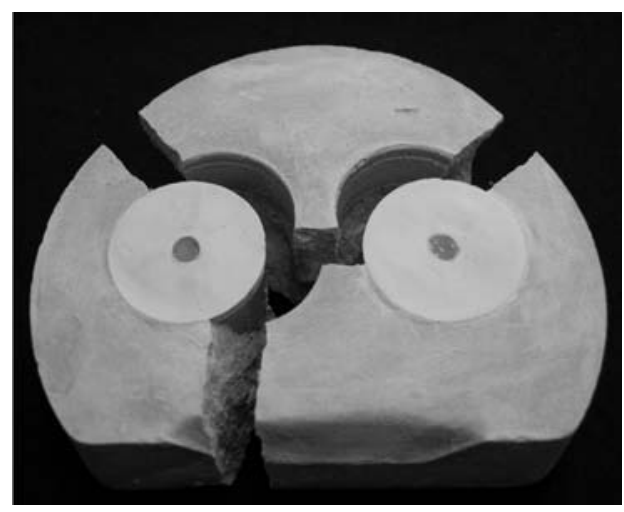

(a)

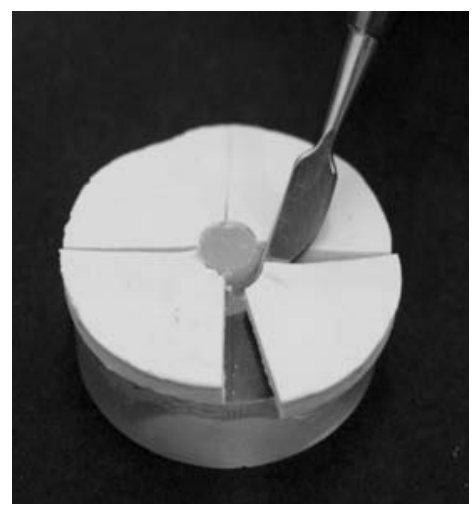

(b)

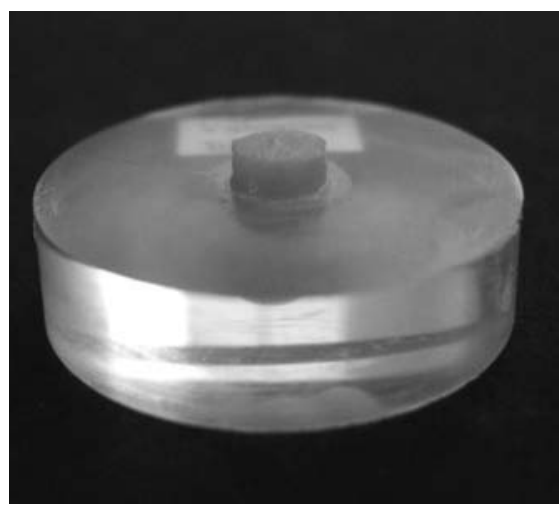

(c)

Figura 7. Desinclusão do espécime: a) desinclusão do gesso; b) remoção do silicone e c) espécime após desinclusão.

nization for Standardization para polímeros de base protética, especificação $1567^{[19]}$.

\section{Ensaio mecânico de cisalhamento}

Cada espécime foi submetido ao ensaio de cisalhamento em uma máquina de ensaios mecânicos EMIC, modelo DL 3000 com célula de carga de 200 kgf (Figura 8a).

Uma matriz metálica foi confeccionada para posicionar o espécime perpendicularmente à força empregada pelo cinzel durante o ensaio de cisalhamento (Figura 4). Essa força na junção dente artificial - resina acrílica para base protética foi aplicada a uma velocidade de $1 \mathrm{~mm} / \mathrm{min}^{[17]}$ até ocorrer a fratura da resina acrílica para base protética (Figura 8b).

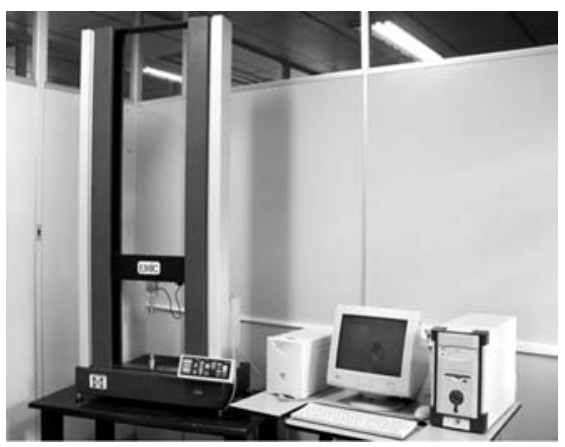

(a)

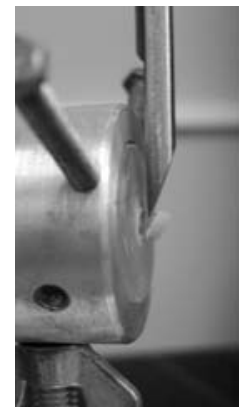

(b)

Figura 8. Ensaio mecânico de cisalhamento: a) máquina universal de ensaios mecânicos (EMIC); e b) espécime fixo na matriz durante o ensaio de cisalhamento.

\section{Resultados e Discussão}

Os dados obtidos foram submetidos ao procedimento estatístico da análise de variância a dois fatores (two way ANOVA) e, posteriormente, foram realizadas comparações múltiplas de médias pelo teste de Tukey em nível de 5\% de significância. Os valores médios de resistência de união para os espécimes polimerizados em todos os Grupos encontramse na Tabela 3.

No presente estudo, verificou-se que, para o mesmo tipo de dente (Figura 9), o grupo polimerizado por meio da energia de microondas (Grupo A) apresentou resistência de união entre dente-resina acrílica estatisticamente superior ao grupo da resina QC 20 (Grupo Q), e semelhante ao grupo da resina Lucitone 550 (Grupo L). Os menores valores de resistência de união observados no Grupo Q em relação aos Grupos A e $\mathrm{L}$ podem ser explicados pelo fato de que o processamento da resina QC 20 utiliza um ciclo de polimerização com tempo

Tabela 3. Média, desvio-padrão (DP) e intervalo de confiança (IC) da resistência de união, em MPa, para todos os grupos.

\begin{tabular}{cccccc}
\hline & \multicolumn{2}{c}{ Biotone } & & \multicolumn{2}{c}{ Trilux } \\
\cline { 2 - 3 } \cline { 5 - 6 } Grupo & média \pm DP & IC & & média \pm DP & IC \\
\hline A & $8,25 \pm 2,00$ & \pm 1.24 & & $10,84 \pm 4,70$ & \pm 2.91 \\
$\mathrm{~L}$ & $7,53 \pm 3,56$ & \pm 2.21 & & $9,39 \pm 2,61$ & \pm 1.62 \\
$\mathrm{Q}$ & $7,46 \pm 2,24$ & \pm 1.39 & & $5,80 \pm 1,71$ & \pm 1.06 \\
\hline
\end{tabular}

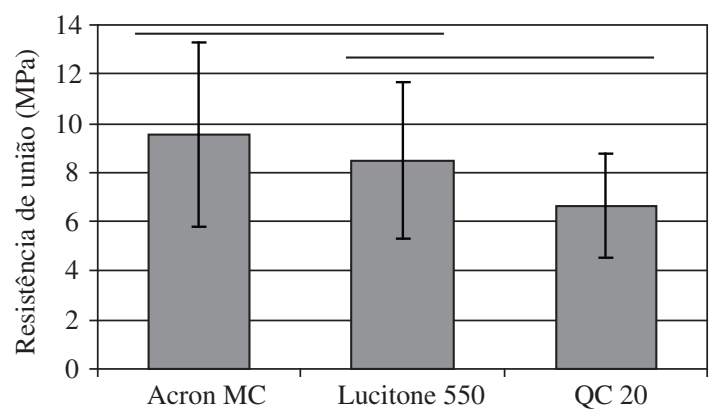

Figura 9. Valores médios da resistência máxima de união para as três resinas acrílicas, para o mesmo tipo de dente. As barras de erro representam os desvios-padrão, e as linhas horizontais indicam semelhança estatística (teste HSD de Tukey, $\alpha=0,05)$. 
reduzido a uma temperatura elevada. Dessa forma, a reação exotérmica de polimerização é acentuada, com a conseqüente evaporação do monômero e a formação de poros ${ }^{[20,21]}$, prejudicando, com isso, a resistência de união.

Outro fator que também deve ser considerado está relacionado à própria composição da resina QC 20 , já que ela contém um sistema de ativação (dimetil-para-toluidina) adicionado ao líquido, semelhante à composição das resinas acrílicas quimicamente ativadas. Esse fato poderia gerar uma pré-polimerização da resina e, portanto, diminuir a quantidade de moléculas de monômero disponíveis para estabelecer a interpenetração das moléculas na interface dente artificial - resina acrílica, prejudicando a união entre esses dois materiais.

Em relação ao grupo onde foi utilizada a resina acrílica Lucitone 550 (Grupo L), os valores de resistência de união obtidos podem ser explicados pelo fato de que o ciclo de polimerização indicado pelo fabricante é mais longo que o da resina QC 20. Isso permite um maior tempo para que ocorra a difusão dos monômeros da resina acrílica da base protética para o interior do polímero dos dentes artificiais, auxiliando na união química entre estes materiais ${ }^{[21]}$.

Já para o Grupo A, onde utilizou-se a resina Acron MC, os trabalhos de Bafile et al. ${ }^{[23]}$, Borges et al. ${ }^{[24]}$, Geerts \& Jooste ${ }^{[6]}$, Paes Jr. et al. ${ }^{[2]}$ e Yamauchi et al..$^{[12]}$, juntamente com o presente estudo, reforçam que a energia de microondas quando utilizada em ciclos com tempo e potência adequados, podem produzir propriedades na resina acrílica semelhantes ao método convencional em banho de água.

O tipo de dente artificial também tem sido avaliado por alguns autores ${ }^{[7,25-27]}$, como fator de influência na resistência de união entre o dente e a resina de base. Alguns dentes artificiais, por apresentarem em sua composição agentes de ligação cruzada teriam, segundo Takahashi et al. ${ }^{[7]}$, um aumento na resistência à fratura, abrasão e ao manchamento. Entretanto, a sua união à resina de base não seria tão efetiva em comparação ao dente artificial de resina acrílica convencional.

Isso pode ser reforçado através do estudo de Clancy \& Boyer ${ }^{[27]}$, que verificaram a influência da composição do dente artificial na resistência de união e obtiveram os maiores resultados entre um dente artificial convencional (Trubyte Bioform) em relação ao dente com agentes de ligação cruzada (Trubyte Bioform IPN) unidos a uma mesma resina acrílica termopolimerizável convencional (Microlon).

Takahashi et al. ${ }^{[7]}$, ao avaliarem os mesmos tipos de dente citados no estudo de Clancy \& Boyer ${ }^{[27]}$, também observaram que o dente de resina acrílica convencional proporcionou maior união aos três tipos de resinas para base protética avaliados. A explicação para isso seria que a adição de agentes de ligação cruzada reduz a quantidade de cadeias poliméricas livres no dente artificial, o que diminui consideravelmente a interação deste com a resina de base.

No presente estudo, embora não tenha sido avaliada a composição do dente artificial, foi possível observar que, para a mesma resina acrílica, a resistência de união entre dente e resina foi semelhante independente do tipo de dente utilizado. Chai et al. ${ }^{[25]}$ avaliaram a influência do tipo de dente na resistência de união, utilizando um dente de resina acrílica convencional (à base de poli metilmetacrilato, Trubyte Bioform) e outro contendo em sua composição agentes de ligação cruzada (Trubyte Bioform IPN), unidos a um mesmo tipo de resina acrílica para base protética. Assim como no presente estudo, os autores observaram que o tipo de dente não afetou a resistência de união.

Diante da proposta apresentada, onde procurou-se verificar a resistência de união entre dois tipos de dente artificiais e três tipos de resina acrílica para base protética, obtivemos resultados que nos permitem indicar a polimerização por meio da energia de microondas como uma alternativa à polimerização da resina acrílica para base protética. O método de polimerização em microondas apresenta não só vantagens no processamento laboratorial, tais como a rapidez e a limpeza nos procedimentos, mas também está de acordo com diversos trabalhos que demonstraram que a utilização da energia de microondas na polimerização da resina não interfere nas suas propriedades, apresentando resultados muitas vezes semelhantes ou superiores aos observados para o ciclo convencional em banho de água quente ${ }^{[6,12,23,24,28,29]}$.

A partir dos resultados, também foi possível concluir que a hipótese de que o tipo de dente, para uma mesma resina, não influencia na resistência de união foi aceita, no entanto, a segunda hipótese do estudo deve ser rejeitada, uma vez que, para o mesmo tipo de dente, o tipo de resina teve influência.

\section{Conclusões}

De acordo com os experimentos realizados, pode-se concluir que:

- Para a mesma resina, a resistência de união entre dente artificial e resina acrílica foi semelhante independentemente do tipo de dente utilizado; e

- Para os dois tipos de dente avaliados (Biotone e Trilux), as resinas Acron MC e Lucitone 550 apresentaram valores de resistência de união entre dente artificial e resina acrílica estatisticamente semelhantes e superiores em relação à resina QC-20.

\section{Agradecimentos}

Ao CNPq/ PIBIC, pelo apoio financeiro e ao Prof. Dr. Raphael Freitas de Souza, pela realização da análise estatística.

\section{Referências Bibliográficas}

1. Kelly, G. B. - J. Prosthet. Dent., 1, n.1-2, p.168-176 (1951).

2. Amin, W.M. - Eur. J. Prosthodont. Restor. Dent., 10, n.2, p.57-61 (2002).

3. Barpal, D.; Curtis, D.A.; Finzen, F.; Perry, J. \& Gansky, S.A. - J. Prosthet. Dent., 80, n.6, p.666-671 (1998). 
4. Cardash, H. S.; Liberman, R. \& Helft, M. - J. Prosthet. Dent, 55, n.4, p.526-528 (1986).

5. Cunningham, J. L. \& Benington, I. C. - J. Dent., 27, n.2, p.129-135 (1999).

6. Geerts, G.\& Jooste, C. H. - J. Prosthet. Dent., 70, n.5, p.406-409 (1993).

7. Takahashi, Y.; Chai, J.; Takahashi, T. \& Habu, T. - Int. J. Prosthodont., 13, n.1, p.59-65 (2000).

8. Spratley, M.H. - J. Prosthet. Dent., 58, n.3, p.389-392 (1987).

9. Patil, S. B.; Naveen, B. H. \& Patil, N. P. - Gerodontology, 23, p. 131-139 (2006).

10. Polyzois, G. L. \& Dahal, J. E. - Eur. J. Prosthodont. Restor. Dent., 2, n.1, p.41-44, (1993).

11. Schneider, R. L., Curtis, E. R. \& Clancy, J. M. S. - J. Prosthet. Dent., 88, n.2, p.145-150 (2002).

12. Yamauchi, M.; Iwahori, M.; Sakai, M.; Koda, T.; Kawano, J. \& Maeno, T. Gifu Shika Gakki Zasshi, 16, p.542-550 (1989).

13. Nishii, M. - J. Osaka Dent. Univ., 2, n.1, p.23-40 (1968).

14. De Clerck, J. P. - J. Prosthet. Dent., 57, n.5, p.650-658 (1987).

15. Ilbay, S.G.; Güvener, S. \& Alkumru, H.N. J. - J. Oral Rehabil., 21, n.1, p.103-109 (1994).

16. Levin, B.; Sanders, J. L. \& Reitz, P. V. - J. Prosthet. Dent., 61, n.3, p.381-383 (1989).

17. International Organization for Standardization. Dentistry: polymer-based crown and bridge materials. ISO 10477: 1992 DAM 1 Draft Amendment. ISO/IC 106/ SC 2 (1995).
18. Pero, A. C. - " Avaliação da influência do método de polimerização e da espessura da base de próteses totais na porosidade da resina acrílica", Dissertação de Mestrado, Universidade Estadual Paulista, Brasil (2005).

19. International Organization for Standardization. Dentistry: denture base polymers. ISO 1567: 1998 (E) Switzerland (1998).

20. Honorez, P.; Catalan, A.; Agnes, U. \& Grimonster, J. - J. Prosthet. Dent.,61, n.4, p.510-517 (1989).

21. Paes Jr., T. J. A.; Marchini, L. \& Kimpara, E.T. - PGR: Pós-Graduação em Revista, São José dos Campos, 2, n.2, p. 36-42 (1999).

22. Vallittu, P. K. - Acta Odontol. Scand., 53, n.2, p.99-104, (1995).

23. Bafile, M.; Graser, G. N.; Myers, M. L. \& Li, E. K. H. - J. Prosthet. Dent., 66, n.2, p.269-274 (1991).

24. Borges, L. H.; Domitti, S. S. \& Consani, S. - Rev. CROMG, 6, n.2, p. 68-77 (2000).

25. Chai, J.; Takahashi, Y.; Takahashi, T. \& Habu, T. - Int. J. Prosthodont.,13, n.2. p.112-116 (2000).

26. Lagouvardos, P. E. \& Polyzois, G. L. - Int. J. Prosthodont., 16, n.5, p.499-504 (2003).

27. Clancy, J. M. S. \& Boyer, D. B. - J. Prosthet. Dent.,61, n.4, p.457-462 (1989).

28. Compagnoni, M. A.; Barbosa, D. B.; Souza, R. F.\& Pero, A. C. - J. Prosthet. Dent., 91, n.3, p.281-285 (2004).

29. Ganzarolli, S. L.; Rached, R. N., Garcia, R. C. M. R. \& Del Bel Cury, A. A. - Pós-Grad. Rev. Fac. Odontol. São José dos Campos, 2, n.2, p.79-86 (1999).

Enviado: $27 / 11 / 06$

Reenviado: 10/03/07

Aceito: 09/04/07 